\title{
Variações espaço-temporais no estoque de sementes do solo na floresta amazônica
}

\author{
Niwton LEAL FILHO ${ }^{1}$, Juliana dos Santos SENA², Gisele Rodrigues dos SANTOS ${ }^{3}$
}

RESUMO

A dispersão eficiente, a longevidade e a capacidade das sementes de permanecer em estado latente a espera de condiçóes adequadas de germinação no banco de sementes do solo da floresta garantem a presença de espécies arbóreas pioneiras nas áreas perturbadas. As variações estacionais e espaciais na densidade e na composiçáo florística do banco de sementes em Florestas Tropicais Úmidas são assuntos ainda pouco compreendidos. Este trabalho verificou a existência de modificaçóes espaço-temporais do banco de sementes presente em áreas de Floresta Tropical úmida localizadas próximas a Manaus, AM. Em cada uma das seis áreas estudadas, foram coletadas 40 amostras circulares de solo superficial (10 cm de diâmetro e $2 \mathrm{~cm}$ de profundidade) ao acaso. Essas amostras foram coletadas a cada dois meses, entre agosto/2004 e junho/2005,. As amostras de solo foram distribuídas em bandejas em casa de vegetação e a emergência das sementes presentes no solo foi acompanhada por 4 meses. Houve uma redução significativa $(\mathrm{H}: 14,09, \mathrm{p}<0,05)$ na densidade média de sementes no solo em junho (início da estação seca) em relação a fevereiro (meio da estação chuvosa). Houve também diferença significativa (H: 188,72, p < 0,05) na densidade média de sementes do solo presente nas diferentes áreas amostradas. Assim como para outras áreas de florestas tropicais, o banco de sementes permanente da floresta foi dominado por espécies pioneiras, principalmente da família Melastomataceae, enquanto as espécies típicas da Floresta Tropical madura foram raras no solo florestal.

PALAVRAS-CHAVE: banco de sementes, Amazônia, Reserva Florestal Adolpho Ducke.

\section{Space-time variations in soil seed stock in an amazon rainforest}

\section{ABSTRACT}

The dispersion efficiency, longevity and the ability of seeds to remain latent waiting for suitable conditions for germination in the forest soil seed bank ensures the presence of pioneer tree species in disturbed areas. The seasonal and spatial variations in the density and floristic composition of the seed bank in tropical rainforests is a subject still little understood. This work verified the existence of spatio-temporal changes of the seed bank present in areas of humid tropical rain forest located near Manaus, Amazonas. In each of the six study areas, 40 circular samples of topsoil $(10 \mathrm{~cm}$ in diameter and $2 \mathrm{~cm}$ deep) were randomly collected every two months, from August 2004 to June/2005. Inside a greenhouse, the collected soil samples were distributed in trays and the emergence of seeds present in the soil was accompanied during four months. There was a significant reduction $(\mathrm{H}: 14.09112, \mathrm{p}<0.05)$ in the mean density of seeds presents in the soil in June (early dry season) compared to February (middle of the rainy season). There was also a significant difference $(\mathrm{H}: 188.7245, \mathrm{p}<0.05)$ in the mean density of soil seeds present in the different samples areas. Similar to other tropical forest areas, the permanent seed bank in the forest was dominated by pioneer species, mainly from the family Melastomataceae, while typical tropical forest species were rare in the forest soil.

KEYWORDS: seed bank, Amazon, Adolpho Ducke Forest Reserve.

\footnotetext{
1 Instituto Nacional de Pesquisas da Amazônia, Campus III - V8, Coordenação de Biodiversidade, Av. André Araújo, 2.936, Petrópolis, Brasil, Cx. Postal 2223 - CEP 69080-971 Manaus, AM, Brasil. E-mail: niwton@inpa.gov.br

2 Instituto Brasileiro do Meio Ambiente e dos Recursos Naturais Renováveis, Rod BR 319, s/n, Km 01, Distrito Industrial, CEP 69075-830, Manaus, AM, Brasil. E-mail: sena_juliana@yahoo.com.br

${ }^{3}$ Instituto Nacional de Pesquisas da Amazônia, Campus III - V8, Coordenação de Biodiversidade, Av. André Araújo, 2.936, Petrópolis, Brasil, Cx. Postal 2223 - CEP 69080-971, Manaus, AM, Brasil. E-mail: gisele.santos@inpa.gov.br
} 


\section{INTRODUÇÃO}

A compreensão dos mecanismos envolvidos na manutenção da estrutura e da biodiversidade da Floresta Tropical úmida é fundamental para seu manejo e conservação, existindo uma concordância generalizada de que dinâmica de ocorrência dos distúrbios naturais relaciona-se estreitamente com as características florísticas e estruturais desta floresta (Pickett e White 1985; Chazdon 2003; Castillo e Stevenson 2010).

Após o distúrbio, a vegetação da Floresta Tropical úmida regenera-se basicamente por três potenciais: o potencial seminal edáfico, representado pelo banco de sementes; o potencial adventício, representado pela chuva de sementes e o potencial vegetativo, representado pelo banco de plântulas e por indivíduos originados da rebrota de partes vegetativas (Alexandre 1982; Martins e Engel 2007). O papel relativo destes potenciais no processo de regeneração depende, no entanto, diretamente do tipo e da intensidade do distúrbio (Connell 1989; Schupp et al. 1989; Denslow e Hartshorn 1994).

O banco de sementes tem sido apontado em vários trabalhos, como preponderante na regeneração de natural das florestas submetida a distúrbios de maior intensidade, como na abertura de grandes clareiras naturais ou abertas durante a exploração da floresta em regime de manejo florestal sustentado (Putz e Appanah 1987; Lawton e Putz 1988; Castillo e Steverson 2010).

Nas florestas tropicais o banco de sementes transitório é formado principalmente por espécies climáxicas que possuem sementes recalcitrantes ou ainda por sementes de outros grupos ecológicos com período de viabilidade curto, enquanto o banco de sementes permanente é formado em geral por espécies pioneiras, com a capacidade de permanecerem viáveis no solo por longos períodos de tempo, em estado de dormência endógena ou latência, a espera de condições adequadas de germinar e estabelecer (Baskin e Baskin,1998; Ferraz et al. 2004; Jankowska-Blaszczuk e Grubb 2006).

O banco de sementes pode ser visto como um sistema dinâmico onde o balanço entre entradas e saídas determina um estoque acumulado de sementes no solo, que varia substancialmente no tempo e no espaço influenciado por fatores bióticos e abióticos (Dalling et al. 1998; Fornara e Dalling 2005; Williams et al. 2005).

O período de viabilidade das sementes no solo varia entre as espécies (Baskin e Baskin 1998; Grombone-Guarantine e Rodrigues 2002; Salazar et al. 2011), podendo variar de semanas a décadas. Por outro lado, a densidade de sementes de determinada espécie no solo é influenciada pela distância de indivíduos co-específicos (Dalling et al. 1998), pela capacidade de resistir a predadores e patógenos, seja pela presença de tegumentos protetores ou substancias com características fungicidas ou bactericidas (Dalling et al. 1998) e pela duração do período de frutificação, (Garwood 1989; Souza e Válio 2001).

A densidade e a composição florística do banco de sementes também variam com os estágios sucessionais da Floresta Tropical, com a redução da densidade total e aumento na representatividade de sementes de espécies arbóreas lenhosas nos estágios mais avançados da vegetação (Saulei e Swaine 1988), entre microsítios dentro da mesma área (Rajan et al. 1995; Costa, 1999; Miranda Neto et al. 2010), com a produtividade dos ecossistemas localizados em diferentes altitudes e estaçóes climáticas distintas influenciando os ciclos fenológicos das espécies (Williams-Linera 1993; Jordano 2000) e ainda pela ocorrência de eventos catastróficos como incêndios (Smith et al. 2000) e inundaçôes (James et al. 2007).

A importância de conhecer a dinâmica espacial e sazonal do banco de sementes explica-se por envolver os processos adaptativos das espécies em resposta aos fatores ambientais e pela influência que estas alteraçóes podem ter no direcionamento da regeneração em diferentes áreas e épocas do ano, podendo afetar a densidade de indivíduos e composição das espécies envolvidas no processo, além de servir de base ao manejo e a conservação de espécies de relevantes interesses ecológicos.

Informaçóes sobre a variação espaço-temporal na composição florística e na densidade do banco de sementes presente sob a Floresta Tropical Úmida na região da Amazônia Central são, de nosso conhecimento, inexistentes. Assim, neste trabalho, além de caracterizar a densidade e a composição do banco de sementes nesta floresta, objetivou-se identificar possíveis variaçóes espaciais e sazonais, avançando na compreensão da dinâmica de regeneração natural da Floresta Tropical Úmida. Considerando os resultados dos trabalhos citados anteriormente, o fato de que a Floresta Tropical Úmida é formada por um mosaico representativo dos diversos estágios de regeneração e da existência de duas estaçôes climáticas distintas em relação a precipitação e a incidência solar, propomos as hipóteses de que exista uma variação espaço-temporal significativa na densidade do banco de sementes desta floresta.

\section{MATERIAL E MÉTODOS}

O estudo foi desenvolvido na Reserva Florestal Adolpho Ducke (RFAD) (02 $53^{\prime} \mathrm{S}$ e $\left.59^{\circ} 58^{\prime} \mathrm{W}\right)$, de propriedade do Instituto Nacional de Pesquisas da Amazônia (INPA). A Reserva possui uma área de 10.000 ha de Floresta Tropical Úmida, localizada no Km 26 da AM-010 (Manaus Amazonas) (Ribeiro et al. 1999) e suas laterais se limitam com sítios e ao sul faz limite com bairros de Manaus. 
A vegetação da Reserva é classificada como Floresta de terra firme, e em uma escala mais detalhada, diferentes habitats podem ser reconhecidos, além da vegetação secundária das bordas e arredores. O relevo é ondulado, com uma variaçáo altitudinal de $80 \mathrm{~m}$ entre os platôs originais e as partes mais baixas. Nos platôs ocorrem solos argilosos, de sedimentos mais antigos e nas partes mais baixas ocorrem solos arenosos mais recentes (Chauvel 1982; Ribeiro et al. 1999).

O clima regional, de acordo com a classificação de Köppen, é Am quente e úmido durante todo o ano. A temperatura média anual é de $27,6{ }^{\circ} \mathrm{C}$, com temperatura média mínima de $23{ }^{\circ} \mathrm{C}$ e média máxima de $31,2^{\circ} \mathrm{C}$. A temperatura pode variar $10^{\circ} \mathrm{C}$ ao dia e $5{ }^{\circ} \mathrm{C}$ entre as diferentes estaçóes do ano. A média anual da precipitação é de $2.478 \mathrm{~mm}$, apresentando duas estaçôes razoavelmente definidas. A estação de menor precipitação ocorre de junho a outubro e a estaçáo chuvosa ocorre de novembro a maio (Ranzani 1980).

O INPA vem desenvolvendo pesquisas de longo prazo utilizando transectos permanentes que acompanham as curvas de nível do terreno $(250$ x $40 \mathrm{~m})$ e estấo distribuídos sistematicamente pela Reserva Ducke (PELD 1998). A vegetaçáo estabelecida nestes transectos encontra-se identificada, o que permitiu traçar inferências sobre a relação entre as espécies estabelecidas nas áreas e as sementes germinadas durante o desenvolvimento de estudos de banco de sementes (Castilho 2004).

Para o estudo do banco de sementes, foram escolhidos seis destes transectos localizados sobre platôs livres de inundaçóes periódicas e com vegetaçáo típicas da Floresta Tropical Úmida da Amazônia central (Figura 1). Em cada área, foram coletadas aleatoriamente 40 amostras circulares de solo superficial com dimensóes de $2 \mathrm{~cm}$ de profundidade e $10 \mathrm{~cm}$ de diâmetro $\left(157,08 \mathrm{~cm}^{3}\right.$ de solo por amostra), totalizando 240 amostras por coleta. A localização das parcelas escolhidas foi a seguinte: parcela 1 (A1) $-2^{\circ} 59.070^{\prime} \mathrm{S}$ e $59^{\circ} 54.665^{\prime} \mathrm{W}$, parcela 2 (A2) $-2^{\circ} 59.037^{\prime} \mathrm{S}$ e $59^{\circ} 55.196^{\prime} \mathrm{W}$, parcela 3 (A3) - 2058.379'S e $59^{\circ} 54.723^{\prime} \mathrm{W}$, parcela 4 (A4) - 2058.403'S e $59^{\circ} 54.731^{\prime} \mathrm{W}$, parcela 5 (A5) $-2^{\circ} 55.614^{\prime} \mathrm{S}$ e $59^{\circ} 57.663^{\prime} \mathrm{W}$ e parcela 6 (A6) $-2^{\circ} 55.559^{\prime} \mathrm{S}$ e $59^{\circ} 53.041^{\prime} \mathrm{W}$.

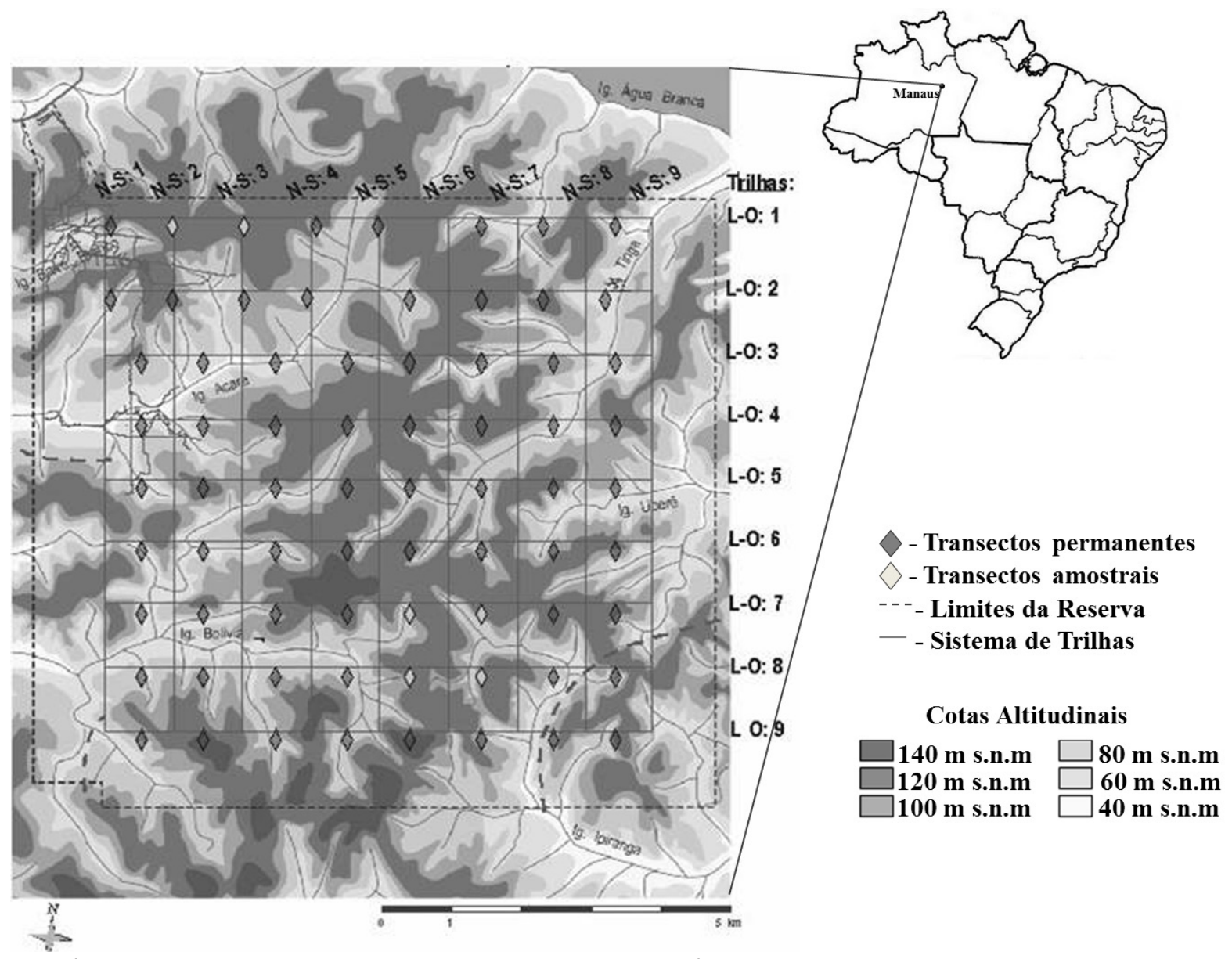

Figura 1 - Mapa da Reserva Ducke (INPA), Manaus, AM, com sistema de trilhas e áreas amostrais. 
Durante a realizaçáo das coletas, a liteira grossa (folhas inteiras ou parcialmente decompostas e galhos acima de 0,5 $\mathrm{cm}$ ) foi removida após a confirmação da ausência de resíduo de solo ou sementes intactas. Assim, as amostras de solo superficial foram coletadas apenas com a liteira mais fina. As amostras foram transportadas em sacos plásticos devidamente identificados. Durante um ano, foram realizadas seis coletas bimestrais (agosto/2004; outubro/2004; dezembro/2004; fevereiro/2005; abril/2005 e junho/2005) durante o período de um ano, incluindo assim os períodos de chuva e seca.

Para contagem e identificação das espécies foi utilizada a técnica de emergência de plântulas sob condiçáo de casa de vegetaçáo, onde as amostras de solo foram colocadas em bandejas plásticas retangulares $(10 \times 27 \times 8 \mathrm{~cm})$. Cada amostra foi espalhada com uma espessura máxima de um centímetro sobre um substrato formado por areia lavada e pó de serragem curtida isento de sementes $(3: 1)$.

Ao emergir, cada plântula foi marcada com canudos plásticos numerados e posteriormente foram identificadas quando possível por morfotipo, espécie e hábito de vida através de consultas a parabotânicos, pesquisadores e literatura especializada (Duke 1965; Ribeiro et al. 1999; Garwood 2009). As contagens e identificaçôes eram realizadas mensalmente e os indivíduos não identificados foram repicados para sacos plásticos individuais para posterior identificação. As espécies tiveram os nomes científicos e suas respectivas famílias atualizados de acordo com o sistema do Angiosperm Phylogeny Group III (APG III 2009).

As amostras foram acompanhadas por um período aproximado de quatro meses, pois segundo Vieira (2004), o maior percentual de sementes germinadas ocorre no primeiro mês, decrescendo até o esgotamento de sementes no solo.

A casa de vegetaçáo, localizada no Campus III (V8) do INPA, em Manaus, onde o trabalho foi conduzido, possui telhado formado por telhas plásticas transparentes, que permitem a passagem de luz e ao mesmo tempo protegem as plântulas do impacto da chuva. As laterais da casa de vegetaçáo são cercadas com sombrite $50 \%$, capaz de permitir a ventilação natural e evitar a entrada de sementes contaminantes.

Durante o acompanhamento da germinação, foram mantidas seis bandejas com substrato composto por areia lavada e serragem (3:1) para servirem como controle de possíveis contaminaçōes externas. As regas foram realizadas diariamente ou conforme a necessidade do momento, dependendo das condiçôes climáticas do dia.

As densidades médias de sementes observadas para as diferentes coletas e áreas foram comparadas utilizando-se o teste náo paramétrico de Kruskal-Wallis (Zar 1996) e quando significativo foi utilizado o teste de comparaçóes múltiplas das médias obtidas nas distintas épocas e áreas. As análises foram executadas com o uso do programa Statistica versão 8.0. A composição e a distribuição de espécies por hábito de vida, segundo definição de Ribeiro et al. (1999) foram discutidas com base nos resultados obtidos pela estatística descritiva. A similaridade da composição florística do banco de sementes no espaço e no tempo foram comparadas utilizando-se o coeficiente de similaridade de Sørensen (Matteucci e Colma 1982).

\section{RESULTADOS}

Comparando a densidade de sementes encontrada no solo superficial coletado em diferentes meses do ano na RFAD (Figura 2), verificou-se que houve diferença significativa ( $\mathrm{H}: 14,09, \mathrm{p}<0,05)$ entre a média observada em fevereiro, densidade de 534 sementes. $\mathrm{m}^{-2}$, em plena estação chuvosa, e junho, 388 sementes. $\mathrm{m}^{-2}$, início da estação seca.

Também houve diferença significativa (H: 188,72, $\mathrm{p}<$ $0,05)$ na densidade de sementes do solo entre as diferentes áreas de platô estudadas. A densidade média nas áreas apresentou uma amplitude de 276 a 811 sementes m-2 ${ }^{2}$ (Figura 3). As áreas 2 e 5 foram as que apresentaram maior densidade

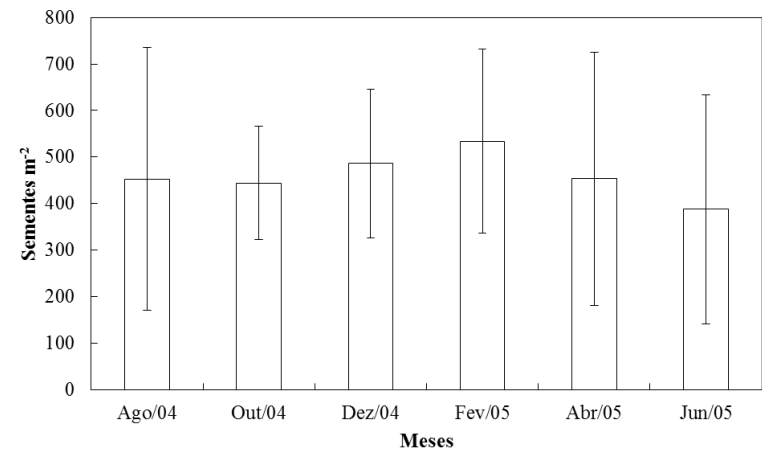

Figura 2 - Densidade média e desvio padrão do número de sementes m-2 germinadas no banco de sementes de áreas de platô da Reserva Florestal Adolpho Ducke, Manaus, AM, em diferentes épocas do ano.

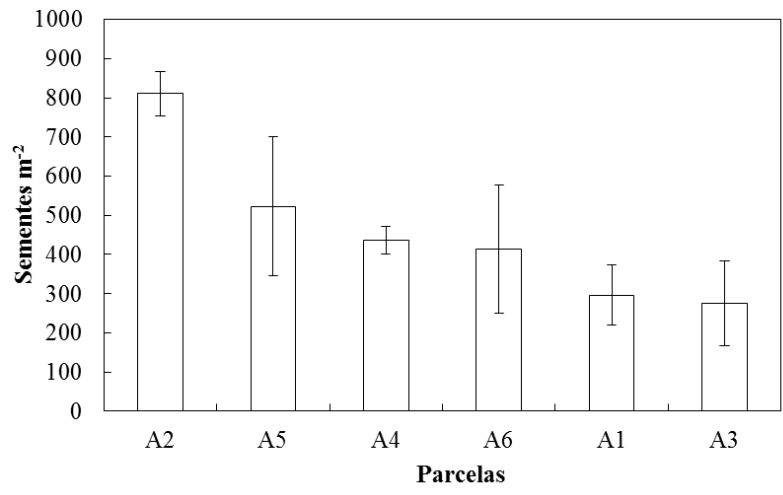

Figura 3 - Densidade média (sementes/ $\mathrm{m}^{2}$ ) de sementes no banco de sementes, de áreas de platô da Reserva Florestal Adolpho Ducke, Manaus, AM, coletadas em diferentes épocas do ano. 
média de sementes, enquanto as áreas 1 e 3 apresentaram as menores.

Como em outras áreas de Floresta Tropical, o banco de sementes da área foi dominado principalmente por espécies pioneiras (Garwood 1989). Das sementes germinadas, $99,65 \%$ pertenceram a este grupo. Em relação à composição florística do banco de sementes, foram identificados um total de 36 famílias e 77 morfotipos ou espécies (Tabela 1). O banco de sementes foi dominado pela família Melastomataceae, representada principalmente pelos gêneros Bellucia spp. e Miconia spp., que representaram $62 \%$ dos indivíduos emergidos. Em seguida predominaram espécies das famílias Urticaceae (Cecropia spp., Coussapoa spp.) com 19\% dos indivíduos germinados e Clusiaceae (Vismia guianensis, Vismia cayannensis, Vismia sp.) com 5\% dos indivíduos germinados no banco de sementes do solo.

Verificou-se que, independentemente da época e da área, Melastomataceae (Bellucia spp), Urticaceae (Cecropia

Tabela 1 - Número de sementes emergidas de espécies, gêneros ou morfotipos no banco de sementes das amostragens de diferentes épocas do ano, em áreas de platô da Reserva Florestal Adolpho Ducke, Manaus, AM

\begin{tabular}{|c|c|c|c|c|c|c|c|c|}
\hline Família & Morfotipo/gênero/espécie & Hábito de vida ${ }^{(\star *)}$ & Ago/04 & Out/04 & Dez/04 & Fev/ 05 & Abr/05 & Jun/ 05 \\
\hline Annonaceae & Rollinia insignis R. E. Fr. & AP & 3 & 1 & 2 & 1 & & 2 \\
\hline \multirow{3}{*}{ Araceae } & Araceae sp. & PAL & & & 1 & & & \\
\hline & Philodendron sp. & EP & 1 & 12 & 18 & 23 & 29 & 11 \\
\hline & Philodendron sp.1 & HE & & & & & 2 & \\
\hline \multirow{4}{*}{ Asteraceae } & Asteraceae sp. & $\mathrm{E}$ & 1 & & & 1 & & \\
\hline & Erechtites hieracifolia (L.) DC. & $\mathrm{E}$ & 3 & 10 & 1 & & 1 & \\
\hline & Mikania sp. & $\mathrm{E}$ & 2 & & & 1 & 1 & 1 \\
\hline & Wendelia paludosa DC. & E & 2 & 7 & & & & \\
\hline Burseraceae & Trattinnickia sp. & AP & & & 1 & 1 & & \\
\hline Cannabaceae & Trema micrantha (L.) Blume & AP & 1 & 1 & 1 & & 2 & \\
\hline Caricaceae & Carica papaya L. & AP & & 1 & & & & \\
\hline Caryophyllaceae & Drymaria cordata A. Grayssp. Cordata & $\mathrm{E}$ & & 5 & & & & \\
\hline Chrysobalanaceae & Couepia sp. & $\mathrm{AC}$ & 1 & & & & & \\
\hline \multirow{3}{*}{ Clusiaceae } & Vismia cayennensis (Jacq.) Pers. & AP & 17 & 18 & 13 & 26 & 16 & 10 \\
\hline & Vismia guianensis (Aubl.) Choisy & AP & 25 & 41 & 26 & 25 & 24 & 34 \\
\hline & Vismia spp. & AP & 1 & 2 & 6 & & & \\
\hline \multirow{2}{*}{ Cyperaceae } & Kyllinga brevifolia Rottb. & $\mathrm{E}$ & & 1 & & & & \\
\hline & Rhynchospora aurea Vahl & $\mathrm{E}$ & & 2 & 8 & & 3 & 6 \\
\hline \multirow{4}{*}{ Desconhecida } & $\mathrm{NI}$ & Desconhecida & 13 & 11 & 6 & 23 & 8 & 6 \\
\hline & NI sp. & $E$ & & 9 & & & 1 & 1 \\
\hline & $\mathrm{Nl}$ sp.1 & EP & & & & 2 & & \\
\hline & $\mathrm{NI} \mathrm{sp} .2$ & Desconhecida & & & & & 6 & \\
\hline Dilleniaceae & Doliocarpus spp. & $\mathrm{L}$ & 2 & 9 & 7 & 7 & 11 & 6 \\
\hline \multirow{4}{*}{ Euphorbiaceae } & Croton lanjouwensis Jabl. & $\mathrm{AP}$ & & 1 & & & & \\
\hline & Euphorbiacea sp. & $\mathrm{AP}$ & & & & & & 1 \\
\hline & Maprounea sp. & $\mathrm{AP}$ & & & & 1 & & 1 \\
\hline & Sapium sp. & $\mathrm{AP}$ & & & 1 & & & \\
\hline Gentianaceae & Irlbachia alata (Aubl.) & $\mathrm{E}$ & & & & 1 & & \\
\hline Goupiaceae & Goupia glabra Aubl. & $\mathrm{AP}$ & 12 & 11 & 15 & 16 & 14 & 19 \\
\hline Humiriaceae & Endopleura sp. & $\mathrm{AC}$ & & 1 & & & & \\
\hline Icacinaceae & Poraqueiba sp. & $\mathrm{AC}$ & 1 & & & 2 & & 4 \\
\hline \multirow{3}{*}{ Lauraceae } & Aniba sp. & $A C$ & & & & 1 & & \\
\hline & Ocotea sp. & $A C$ & & 1 & 1 & & & \\
\hline & Ocotea sp.1 & AP & & & & 1 & & \\
\hline Lecythidaceae & Eschweilera sp. & $\mathrm{AC}$ & & & & 1 & & \\
\hline Leguminosae & Dinizia excelsa Ducke & $A C$ & & & & & 2 & \\
\hline
\end{tabular}




\begin{tabular}{|c|c|c|c|c|c|c|c|c|}
\hline Família & Morfotipo/gênero/espécie & Hábito de vida ${ }^{(\star *)}$ & Ago/04 & Out/04 & Dez/04 & $\mathrm{Fev} / 05$ & Abr/05 & Jun/ 05 \\
\hline \multirow{2}{*}{ (Mimosoideae) } & Enterolobium sp. & $A C$ & & 3 & & & & \\
\hline & Mimosa guilandinae (DC.) & L & & 1 & & 1 & & 2 \\
\hline \multirow{3}{*}{ Malpighiaceae } & Byrsonima duckeana W. R. Anderson & AP & 1 & & & & 1 & 2 \\
\hline & Byrsonima sp.1 & AP & 2 & & 2 & & & 1 \\
\hline & Byrsonima sp.2 & AP & & 1 & & & & \\
\hline Marantaceae & Ischnosiphon sp. & $\mathrm{E}$ & & 1 & & & & \\
\hline \multirow{10}{*}{ Melastomataceae } & Bellucia sp. & AP & 57 & 43 & 44 & 73 & 35 & 42 \\
\hline & Melastomatacea spp..$\left.^{*}\right)$ & ARB & 435 & 320 & 496 & 531 & 422 & 406 \\
\hline & Tococa spp. & ARB & 27 & 36 & 11 & 18 & 17 & 3 \\
\hline & Miconia spp. & ARB & 5 & 13 & 1 & 9 & 12 & 1 \\
\hline & Miconia cf. regelii Cogn. & AP & 27 & 27 & 14 & 14 & 53 & 3 \\
\hline & Miconia sp.2 & ARB & 1 & & & & & \\
\hline & Miconia sp.3 & ARB & 4 & 3 & & & & \\
\hline & Miconia sp.4 & ARB & 1 & & & & & \\
\hline & Miconia sp.5 & ARB & 1 & & & & & \\
\hline & Miconia sp.6 & ARB & 1 & & & & & \\
\hline Memecylaceae & Mouriri sp. & $\mathrm{AP}$ & & & & 1 & & \\
\hline Moraceae & Ficus sp. & AP & & 6 & & 4 & 4 & 2 \\
\hline Ochnaceae & Cespedesia sp. & $\mathrm{AP}$ & & 5 & 3 & & 1 & 1 \\
\hline Onagraceae & Ludwigia hyssopifolia (G.Don f.) Exell & $\mathrm{E}$ & 2 & & & & & \\
\hline Passifloraceae & Passiflora auriculata H.B.K. & $\mathrm{L}$ & & & 4 & & & \\
\hline \multirow{3}{*}{ Piperaceae } & Piper aduncum L. & ARB & 1 & 1 & & & & \\
\hline & Piper spp. & ARB & & 1 & 2 & & 3 & 3 \\
\hline & Piper sp.1 & ARB & & & 10 & 3 & & \\
\hline \multirow{3}{*}{ Poaceae } & Brachiaria sp. & $\mathrm{E}$ & & & 2 & & 2 & \\
\hline & Paspalum sp. & $\mathrm{E}$ & & & 1 & & & \\
\hline & Poacea spp. & $\mathrm{E}$ & 19 & 19 & 19 & 4 & & 4 \\
\hline \multirow{4}{*}{ Rubiaceae } & Palicourea sp. & AP & & & & 1 & 1 & \\
\hline & Psychotria sp. & ARB & & & & 1 & 1 & \\
\hline & Rubiacea spp. & $E$ & 5 & 7 & & & & 4 \\
\hline & Sabicea amazonensis Wernh. & $\mathrm{L}$ & 3 & 9 & 8 & 11 & 4 & 6 \\
\hline \multirow{3}{*}{ Salicaceae } & Casearia arborea (Rich.) Urb. & AP & & 1 & & & & \\
\hline & Casearia sp. & AP & 1 & 2 & 3 & & & \\
\hline & Laetia procera (Poepp.) Eichler & $\mathrm{AP}$ & 10 & 3 & 6 & 8 & 4 & 7 \\
\hline Siparunaceae & Siparuna sp. & ARB & 1 & & 1 & & & \\
\hline Smilacaceae & Smilax sp. & L & & 1 & & & & \\
\hline \multirow{5}{*}{ Solanaceae } & Solanum rugosum Dunal & ARB & 2 & 5 & 5 & 1 & 2 & 1 \\
\hline & Solanum spp. & ARB & 5 & 4 & 8 & 7 & 5 & 1 \\
\hline & Solanum sp.1 & ARB & 1 & & & & & \\
\hline & Solanum sp.2 & $A B R$ & & 1 & 1 & & & 3 \\
\hline & Solanum stramoniifolium Jacq. & ARB & 2 & 3 & 1 & 6 & 8 & \\
\hline \multirow{4}{*}{ Urticaceae } & Cecropia c. f. purpuracens C. C. Berg & AP & 3 & 24 & 17 & 7 & 13 & 3 \\
\hline & Cecropia cf. concolorWilld. & AP & 2 & 1 & 1 & 2 & 4 & 3 \\
\hline & Cecropia c. f. sciadophylla Mart & AP & 121 & 93 & 124 & 141 & 124 & 114 \\
\hline & Coussapoa spp. & $\mathrm{HE}$ & 28 & 59 & 25 & 31 & 18 & 16 \\
\hline Total geral & & & 853 & 837 & 916 & 1007 & 854 & 730 \\
\hline
\end{tabular}

(*) Nos morfotipos identificados como Melastomatacea spp., Araceae sp. e Asteraceae sp. estão incluídos indivíduos somente identificados a nível de família .

(**) AP: árvore pioneira, AC: árvore clímax, ARB: arbusto, PAL: palmeira, HE: hemiepifita, EP: epífita, E: erva, L: liana. 
spp.) e Clusiaceae (Vismia spp) foram as principais famílias no banco de sementes. Observou-se também a presença de espécies pioneiras de valor comercial como a Goupia glabra (Goupiaceae) com 1,7\% dos indivíduos germinados e a Laetia procera (Salicaceae com 0,7\% dos indivíduos germinados).

As espécies Miconia spp. e Bellucia spp (Melastomataceae), Cecropia spp. e Coussapoa spp (Urticaceae), Vismia guianensis, Vismia cayannensis e Vismia sp. (Clusiaceae), Solanum rugosum e Solanum stramoniifolium (Solanaceae), Doliocarpus spp. (Dilleniaceae), Sabicea amazonica (Rubiaceae) e Ficus spp. (Moraceae) formam o banco de sementes permanente, isto é, foram encontradas no solo desta floresta em todas as áreas e em todos os meses estudados. As espécies arbóreas climáxicas, Enterolobium sp. (Mimosoideae), Ocotea sp.1. e Ocotea sp.2 (Lauraceae), Endopleura sp. (Humiriaceae), Eschweilera sp. (Lecythidaceae), Couepia sp. (Chrysobalanaceae) e Dinisia excelsa (Mimosoideae), formam o banco de sementes temporário (Tabela 1 ).

Os resultados para os índices de similaridade de Sørensen entre meses e áreas (Tabela 2) indicam que há uma homogeneidade no tempo e no espaço em relação à composição florística presente no banco de sementes com índices nunca inferiores a 0,75 .

O hábito de vida predominante no banco de sementes do solo, na RFAD, foi arbustiva $(55,46 \%)$, representada principalmente por indivíduos da família Melastomataceae. Esta família também apresenta espécies pioneiras arbóreas bastante comuns nesta região, como por exemplo, a goiaba de anta (Bellucia spp.) e a tinteira (Miconia cf. regelii). Em seguida, predominaram as arbóreas pioneiras (Cecropia spp., Vismia spp., Goupia glabra, Laetia procera) (32,73\%). Outras

Tabela 2- Matriz do índice de similaridade florística de Sørensen da comunidade de espécies emergidas do banco de sementes em diferentes datas e áreas na Reserva Florestal Adolpho Ducke, Manaus, AM.

\begin{tabular}{lllllll}
\hline Área & A1 & A2 & A3 & A4 & A5 & A6 \\
\hline A1 & 1,00 & & & & & \\
A2 & 0,83 & 1,00 & & & & \\
A3 & 0,80 & 0,77 & 1,00 & & & \\
A4 & 0,96 & 0,88 & 0,86 & 1,00 & & \\
A5 & 0,88 & 0,81 & 0,79 & 0,92 & 1,00 & \\
A6 & 0,90 & 0,85 & 0,82 & 0,95 & 0,87 & 1,00 \\
\hline Tempo & Ago/04 & $\mathbf{0 u t} / \mathbf{0 4}$ & Dez/04 & Fev/05 & Abr/05 & Jun/05 \\
\hline Ago/04 & 1,00 & & & & & \\
\hline Out/04 & 0,78 & 1,00 & & & & \\
Dez/04 & 0,84 & 0,82 & 1,00 & & & \\
\hline Fev/05 & 0,79 & 0,75 & 0,82 & 1,00 & & \\
Abr/05 & 0,83 & 0,80 & 0,88 & 0,84 & 1,00 & \\
\hline Jun/05 & 0,85 & 0,82 & 0,89 & 0,86 & 0,91 & 1,00
\end{tabular}

formas de vida encontradas no banco de sementes em menor número foram: ervas $(3,02 \%)$, epífitas $(1,83 \%)$, hemiepífitas (3,46\%), lianas (1,75\%), arbóreas clímax $(0,35 \%)$ e alguns indivíduos não puderam ser identificados (1,40\%) (Figura 4).

\section{DISCUSSÃO}

Outros trabalhos desenvolvidos em distintos ecossistemas encontraram resultados similares quando estudaram a influência da sazonalidade na dinâmica do banco de sementes. $\mathrm{Na}$ Floresta Estacional Semi-decidual em Campinas, SP, observou-se maior densidade de sementes no solo durante o período das chuvas (Grombone-Guaratini e Rodrigues 2002). Na caatinga nordestina, a densidade de sementes no solo superficial foi maior no fim da estação chuvosa quando comparada com o final da estação seca e a densidade diferiu também entre os diversos micro-sítios estudados, áreas ciliar de baixa inclinaçáo próxima de riacho intermitente, áreas planas afastadas até 150 metros da margem do riacho e áreas de afloramento rochoso que ocorrem isoladas no terreno plano (Santos et al. 2010). Entretanto, um estudo do banco de sementes de florestas de galeria no cerrado mineiro não apresentou diferenças entre a estação chuvosa e a seca, assim como entre os micro-sítios estudados, área denominada dique, localizada às margens do corpo d'água e formada por deposição de sedimentos fluviais, floresta ciliar, localizada na depressão adjacente ao dique e a borda da floresta, definida como a faixa de dez metros de largura estabelecida no limite da formação florestal com as formaçóes campestres (PereiraDiniz e Ranal 2006).

A maior densidade de sementes emergidas em fevereiro foi influenciada principalmente, pela grande quantidade de sementes da família Melastomataceae encontradas no solo neste período (Tabela 1). Observou-se também que neste mesmo período, houve um aumento na quantidade de sementes de espécies pertencentes à família Urticaceae. Diferenças nas características de dispersão e de dormência das sementes influenciam a variação espacial e temporal na composiçáo do banco de sementes (Almeida-Cortez, 2004).

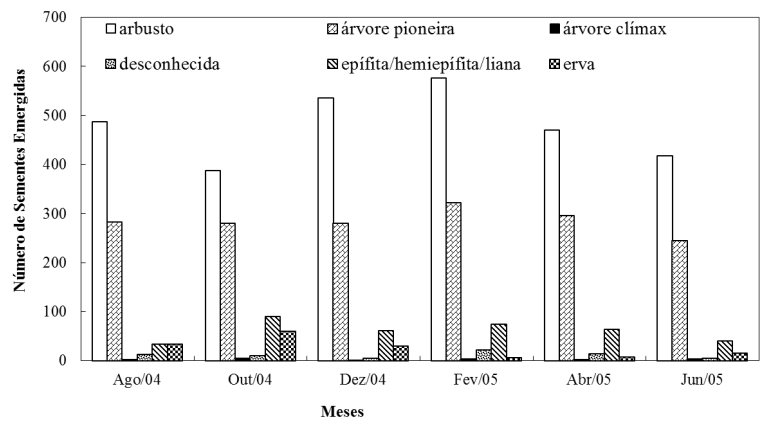

Figura 4 - Formas de vida presentes no banco de sementes de áreas de platô da Reserva Florestal Adolpho Ducke, Manaus, AM, em diferentes épocas do ano. 
As diferenças observadas entre as épocas do ano, estação seca e chuvosa, podem resultar da produção de sementes de espécies pioneiras e clímáxicas, que na região de Manaus concentra-se na estação úmida do ano (Alencar 1994; Bentos 2006). Isto sugere a possibilidade de diferentes caminhos sucessionais em grandes clareiras abertas na floresta em estaçóes distintas do ano, já que o banco de sementes é o principal mecanismo de regeneração na fase inicial de estabelecimento de pioneiras nestas áreas (Grombone-Guaratini e Rodrigues 2002).

Em relação a diferença nas densidades de sementes no solo das áreas estudadas (Figura 3), nota-se que estes resultados estáo dentro da amplitude esperada para Florestas Tropicais maduras, que apresentam uma média de 384 sementes.m- ${ }^{2}$, enquanto a amplitude observada entre Florestas Tropicais Úmidas Neotropicais varia entre 60 e 2340 sementes $\mathrm{m}^{-2}$ (Garwood 1989). A variação existente na densidade de sementes encontrada entre as diferentes áreas pode ser explicada pelo histórico de distúrbios naturais ocorridos anteriormente aos estudos desenvolvidos nestas áreas, como alagamentos temporários, deslizamentos de solo e a presença de grandes clareiras naturais no passado, afetando a composição florística da área, pois, clareiras grandes abertas na floresta, são colonizadas principalmente por espécies pioneiras capazes de produzir um grande número de sementes (Castillo e Stevenson 2010).

A ocorrência no passado de grandes clareiras naquelas áreas, é sugerida pela observaçáo da presença de indivíduos adultos de Cecropia sp. na área 2, que podem enriquecer o banco de sementes da área por sua elevada produção de sementes pequenas e capazes de manter-se latentes no solo por longo período de tempo (Holthuijzen e Boerboom 1982).

A homogeneidade no tempo e no espaço em relação a composiçáo florística no banco de sementes, explica-se pela elevada proporçáo de sementes no solo originada de um grupo restrito de espécies pioneiras que se dispersam eficientemente e que participam do banco de sementes permanente do solo desta floresta (Garwood 1989; Dalling et al. 1998; Grombone-Guaratini e Rodrigues 2002). Todas as famílias predominantes no banco de sementes da RFAD são comuns e abundantes na vegetação de áreas secundárias na regiāo de Manaus.

Baider et al. (1999), estudando o banco de sementes de uma Floresta Atlântica Montana, em São Paulo, verificaram que após a abertura de clareiras naturais a família Melastomataceae, contribuiu com o principal grupo de árvores e arbustos pioneiros na colonizaçáo de clareiras desta floresta ( $88,2 \%$ dos indivíduos). O grupo das pioneiras representou 98\% dos indivíduos amostrados, notando-se a ausência de Urticaceae nesta regiáo.
Araújo et al. (2001), estudando o banco de sementes de florestas em diferentes estágios sucessionais (idades de 6, 17 e 30 anos) na região do baixo rio Guamá - Amazônia oriental, encontraram em todas as áreas a espécie arbórea Miconia serialis (Melastomataceae), que representou mais de $40 \%$ do banco de sementes das florestas estudadas.

\section{CONCLUSÕES}

A densidade média de sementes germinadas nas áreas estudadas está dentro da amplitude encontrada para as Florestas Tropicais úmidas maduras localizadas nos Neotrópicos. Assim como outras áreas de Floresta Tropical, o banco de sementes das áreas de platô da RFAD pode apresentar sementes de espécies climáxicas de forma efêmera, entretanto, é dominado por espécies pioneiras. A composição florística mostrou uma dominância da família Melastomataceae. As hipóteses da existência de variação espaço-temporal no banco de sementes nesta floresta foram confirmadas.

A diferença na densidade do banco de sementes observada entre a época das chuvas e o início da época seca pode resultar da maior produção de sementes das espécies regionais que ocorre principalmente na época chuvosa. A presença de um banco de sementes abundante e rico em espécies durante o ano estudado indica que esta floresta apresenta um grau de resiliência elevada no que se refere ao potencial seminal edáfico, onde os mecanismos de dispersão das espécies pioneiras estariam atuando para manter um número significativo de propágulos no solo no decorrer do ano em todas as áreas estudadas, garantindo assim o processo de regeneração florestal. Áreas em fase de reconstruçáo da floresta, que ocorrem em clareiras antigas e são caracterizadas pela presença de indivíduos adultos de espécies arbóreas pioneiras, tendem a apresentar maior densidade de sementes encontradas no solo, pois as espécies pioneiras maduras estabelecidas nas áreas enriquecem o banco de sementes local.

\section{BIBLIOGRAFIA CITADA}

Alencar, J.C. 1994. Fenologia de cinco espécies arbóreas tropicais de Sapotaceae correlacionada a variáveis climáticas na Reserva Ducke, Manaus, AM. Acta Amazonica 24: 161-182.

Alexandre, D.Y. 1982. Aspects de la régénération naturelle en fôret dense de Côte d'Ivore. Candollea 37: 579-588.

Almeida-Cortez, J.S. 2004. Dispersão e banco de sementes. In: Ferreira, A.G.; Borguetti, F (Ed.). Germinação: do básico ao aplicado. Artmed, Porto Alegre, Rio Grande do Sul, p. 227-235.

APG III - Angiosperm Phylogeny Group III. 2009. An update of the Angiosperm Phylogeny Group classification for the orders and families of flowering plants. Botanical Journal of the Linnean Society, 161: 105-121.

Araújo, M.M.; Oliveira, F.A.; Vieira, C.G.; Barros, P.L.C.; Lima, C.A.T. 2001. Densidade e composição florística do banco de 
sementes do solo de florestas sucessionais na região do baixo rio Guamá, Amazônia Oriental. Scientia Forestalis 59: 115-130.

Baider, C.; Tabarelli, M.; Montovani, W. 1999. O banco de sementes de um trecho de Floresta Atlântica de Montana (Sáo Paulo, Brasil). Revista Brasileira de Biologia 59: 319-328.

Baskin C.C; Baskin J.M. 1998. Seeds: ecology, biogeography and evolution of dormancy and germination. Academic Press, San Diego. 230p.

Bentos, T.V. 2006. Estratégias reprodutivas de espécies pioneiras na Amazônia Central: fenologia e sucesso no estabelecimento de plantas. Dissertação de Mestrado. INPA/UFAM, Manaus, AM. 93p.

Castilho, C.V. 2004. Variação espacial e temporal da biomassa arbórea viva em $64 \mathrm{~km}^{2}$ de floresta de terra firme na Amazônia Central. Tese de Doutorado. INPA/UFAM, Manaus, AM. 72p.

Castillo, L.S; Stevenson, P.R. 2010. Relative importance of seed-bank and post-disturbance seed dispersal on early gap regeneration in a Colombian Amazon Forest. Biotropica 42: 488-492.

Chauvel, A. 1982. Os latossolos amarelos álicos argilosos dentro dos ecossistemas das bacias experimentais do INPA e da regiấo vizinha. Acta Amazonica 12: 47-60.

Chazdon, R.L. 2003. Tropical forest recovery: legacies of human impact and natural disturbances. Perspectives in Plant Ecology, Evolution and Systematics 6: 51-71

Connell, J.H. 1989. Some processes affecting the species composition in forest gaps. Ecology 70: 560-562.

Costa, J.R. 1999. Caracterização dos bancos de sementes de diferentes sistemas de uso da terra na região de Manaus, AM. Dissertaçáo de Mestrado, INPA/UFAM, Manaus, AM. 95p.

Dalling, J.W.; Swaine, M.D. e Garwood, N. 1998. Dispersal patterns and seed bank dynamics of pioneer trees in moist tropical forest. Ecology 79: 564-578.

Denslow, J.S.; Hartshorn, G.S. 1994. Tree-fall gap environments and forest dynamic process. In: McDade, L.; Bawa, K.S.; Hespenheide, H.A.; Hartshorn, G.S. (Ed.). La Selva-Ecology and Natural History of a Neotropical Rain Forest. The University of Chicago Press, Chicago, p.120-127.

Duke, J.A. 1965. Keys for Identification of Seedlings of Some Prominent Woody Species in Eight Forest Types in Puerto Rico. Annals of the Missouri Botanical Garden, 52: 314-350.

Ferraz, I.D.K; Leal Filho, N; Imakawa, A.M; Varela, V.P; PińaRodrigues, F.C.M. 2004. Características básicas para um grupamento ecológico prelimonar de espécies madeireiras da floresta de terra firme da Amazônia Central. Acta Amazonica 34: 621-633.

Fornara, D.A.; Dalling, J.W. 2005. Seed bank dynamics in five Panamanian forests. Journal of Tropical Ecology 21: 223-226.

Garwood, N.C. 1989. Tropical soil seed banks: A review. In: M.A. Leck; V.T. Parker e R.L Simpson (Ed.) Ecology of Soil Seed Banks. Academic Press, San Diego, California. pp. 149-209.

Garwood, N.C. 2009. Seedlings of Barro Colorado Island and the Neotropics. Ithaca, NY: Comstock Pub. Associates/Cornell University Press. 645p.
Grombone-Guaratini, M.T; Rodrigues, R.R. 2002. Seed bank and seed rain in a seasonal semi-deciduous Forest in south-eastern Brazil. Journal of Tropical Ecology 18: 759-774.

Holthuijzen, A.M.A.; Boerboom, J.H.A. 1982. The Cecropia seedbank in the surinam lowland rain forest. Biotropica 14 62-68.

James, C.S; Capon, S.J; White, M.G; Rayburg, S.C; Thoms, M.C. 2007. Spatial variability of the soil seed bank in a heterogeneous ephemeral wetland system in semi-arid Australia. Plant Ecolology 190: 205-217.

Jankowska-Blaszczuk, M.; Grubb, P.J. 2006. Changing perspectives on the role of the soil seed bank in northern temperate deciduous forests and in tropical lowland rain forests: parallels and contrasts. Perspectives in Plant Ecology, Evolution and Systematics 8: 3-21.

Jordano, P. 2000. Fruits and frugivory. In: Fenner, M. (Ed.). Seeds: the ecology of regeneration in plant communities. Commonwealth Agricultural Bureau International, Wallingford, UK. p.125-166.

Lawton, R.O.; Putz, F.E. 1988. Natural disturbance and gap-phase regeneration in a wind-exposed tropical cloud forest. Ecology 69: 764-777.

Martins, A.M; Engel, V.L. 2007. Soil seed banks in tropical forest fragments with different disturbance histories in southeastern Brazil. Ecological Engineering 31: 165-174.

Matteucci, S.D.; Colma, A. 1982. Metodologia para el estudio de la vegetación. Washington: Secretaria geral de la Organización de los Estados Americanos -Programa Regional de Desarrolo Científico y Tecnológico, 169p.

Miranda Neto, A.; Kunz, S.H.; Martins, S.V.; Silva, K.A.; Silva, D.A. 2010. Transposiçáo do banco de sementes do solo como metodologia de restauraçáo florestal de pastagem abandonada em Viçosa, MG. Revista Árvore 34: 1035-1043.

PELD - Projeto Ecológico de Longa Duração/CNPq-INPA. 1998. Disponível em <http://peld.inpa.gov.br/>. Acesso em $18 / 01 / 2012$

Pereira-Diniz, S.G; Ranal, M.A. 2006. Germinable soil seed bank of a gallery forest in Brazilian Cerrado. Plant Ecology 183: 337 -348.

Pickett, S.T.A; White, P.S. 1985. The ecology of natural disturbance as patch dynamics. Academic Press, New York. 472p.

Putz, F.E; Appanah, S. 1987. Buried seeds, Newly dispersed seeds, and the dynamics of a lowland forest in Malaysia. Biotropica 19: $326-333$

Rajan, P; Sundarapandian, SM.; Chandrasekaran, S.; Swamy, P.S. 1995. Variation in soil seed bank at different microsites in a deciduous forest near Madurai, south India. International Journal of Ecology and Environmental Sciences 21: 263-272.

Ranzani, G. 1980. Identificação e caracterização de alguns solos da Estação de Silvicultura Tropical. Acta Amazonica 10: 7-41.

Ribeiro, J.E.L.S.; Hopkins, M.J.G.; Vicentini, A.; Sothers, C.A.; Costa, M.A.D.; Martins, L.H.P.; Lohmann, L.G.; Assunção, P.A.C.L.; Pereira, E.C.; Silva, C.F.; Mesquita, M.R.; Procópio, L.C. 1999. Flora da Reserva Ducke: guia de identificaçâo das plantas vasculares de uma floresta de terra firme na Amazônia Central. INPA/DFID, Manaus. 816 p. 
Salazar, A; Goldstein, G; Franco, A.C; Miralles-Wilhelm, F. 2011. Timing of seed dispersal and dormancy, rather than persistent soil seed-banks, control seedling recruitment of woody plants in Neotropical savannas. Seed Science Research 21: 103-116.

Santos, D.M; Silva, K.A; Santos, J.M.F.F; Lopes, C.G.R; Pimentel, R.M.M; Araújo, E.L. 2010. Variaçáo espaço-temporal do banco de sementes em uma área de floresta tropical seca (caatinga) Pernambuco. Revista de Geografia, 27: 234-253.

Saulei, S.M.; Swaine, M.D. 1988. Rain forest seed dynamics during succession at Gogol, Papua New Guinea. Journal of Ecology 76: 1133-1152.

Schupp, E.W.; Howe, H.F.; Augspurger, C.K. e Levey, D.J. 1989. Arrival and survival in tropical treefall gaps. Ecology 70: 562-564.

Smith, M.A.; Loneragan, W.A.; Grant, C.D.; Koch, J.M. 2000. Effect of fire on the top soil seed banks of rehabilitated bauxite mine sites in the Jarrah forest of western Australia. Ecological Manageament and Restoration 1: 50-60.
Souza, R.P; Válio, I.F.M. 2001. Seed size, seed germination, and seedling survival of brazilian tropical tress species differing in successional status. Biotropica 33: 447-457.

Vieira, D.C.M. 2004. Chuva de sementes, banco de sementes e regeneração natural sob três espécies de início de sucessão em uma área restaurada em Iracemápolis (SP). Dissertaçâo de Mestrado, ESALQ/USP, Piracicaba, SP. 87p.

Williams, P.R; Congdon, R.A.; Grice, A.C.; Clarke; P.J. 2005. Germinable soil seed banks in a tropical savanna: seasonal dynamics and effects of fire. Austral Ecology 30: 79-90.

Williams-Linera, G. 1993. Soil seed banks in four lower montane foresta of Mexico. Journal of Tropical Ecology, 9: 321-337.

Zar, J.H. 1996. Biostatistical Analyses. 3.ed. Prentice-Hall, Inc. New Jersey. 920p.

Recebido em: 19/01/2012

Aceito em: 30/04/2012 